\title{
The Rationale for Photobiomodulation Therapy of Vaginal Tissue for Treatment of Genitourinary Syndrome of Menopause: An Analysis of Its Mechanism of Action, and Current Clinical Outcomes
}

\author{
Raymond J. Lanzafame, MD, MBA, FACS, ${ }^{1}$ Sarah de la Torre, MD, ${ }^{2}$ and Gustavo H. Leibaschoff, MD ${ }^{3}$
}

\begin{abstract}
Objective: Light, particularly in the visible to far-infrared spectrum, has been applied to the female genital tract with lasers and other devices for nearly 50 years. These have included procedures on both normal and neoplastic tissues, management of condylomata, endometriosis, and menometrorrhagia, and, more recently, a number of fractional laser devices have been applied for the management of genitourinary syndrome of menopause (GSM) and stress urinary incontinence (SUI), and to achieve so-called vaginal rejuvenation. Photobiomodulation therapy (PBMT) has been proposed as an alternative for use in managing GSM and SUI.

Methods: This article reviews the biological basis, symptoms, and management of GSM, and investigates the current status and rationale for the use of PBMT.

Results and conclusions: Based on the preliminary evidence available, PBMT is safe and appears to be efficacious in treating GSM.

Keywords: womens' health, genitourinary syndrome of menopause, vagina, tissue regeneration and healing, photobiomodulation, collagen, stress urinary incontinence
\end{abstract}

\section{Introduction}

B OTH THE PUBLIC and the medical community at large are becoming more aware and vocal regarding women's health and related issues. This has included frank discussions of self-awareness and wellness, intimacy, body image ideals, aging and antiaging strategies, as well as cancer prevention and treatment, among other health issues.

Light, particularly in the visible to far-infrared spectrum, has been applied to the female genital tract with lasers and other devices to diagnose and/or treat various diseases for nearly 50 years. These have included procedures on both normal and neoplastic tissues, ablation and excision of condylomata, endometriosis lesion excision and vaporization, and the management of menometrorrhagia and other conditions.
More recently, a number of fractional laser devices have been applied for the management of genitourinary syndrome of menopause (GSM) and stress urinary incontinence (SUI), and to achieve so-called vaginal rejuvenation. The Food and Drug Administration (FDA) has noted that despite the use of various devices for GSM and SUI, it has not approved any devices specifically for these indications and cites adverse events and poor outcomes after use of these technologies. ${ }^{1,2}$

Photobiomodulation therapy (PBMT) has been proposed as an alternative for use in managing GSM and SUI. This article reviews the biological basis, symptoms, and management of GSM, and investigates the current status and rationale for the use of PBMT to treat these conditions. The characteristics of the devices in current use and available clinical and histologic data are presented.

\footnotetext{
${ }^{1}$ Raymond J. Lanzafame, MD PLLC, Rochester, New York.

${ }^{2}$ Seattle OB/GYN Group, Seattle, Washington.

${ }^{3}$ International Consultants in Aesthetic Medicine, Dallas, Texas.

(c) Raymond J. Lanzafame, et al., 2019; Published by Mary Ann Liebert, Inc. This Open Access article is distributed under the terms of the Creative Commons License (http://creativecommons.org/licenses/by/4.0), which permits unrestricted use, distribution, and reproduction in any medium, provided the original work is properly credited.
} 


\section{Genitourinary Syndrome of Menopause}

The vaginal epithelium undergoes changes in response to the level of circulating estrogens during the female reproductive years. Circulating estrogen levels show an affected reduction when menopause occurs. The lack of estrogen induces several modifications of the genital tissues that correlate with the beginning of systematic negative effects. Significant cytological transformations follow estrogen reduction, including the proliferation of connective tissue, the fragmentation of elastin, and collagen hyalinization. These changes may result in a condition described as vulvovaginal atrophy (VVA). ${ }^{3}$

Estrogen receptors have been identified in the pelvic floor musculature and tissues and these are susceptible to estrogen deprivation as occurs in menopause. ${ }^{3-7}$ Vaginal wall connective tissue components, including collagen, elastin, and smooth muscle, all degenerate as a result of estrogen deprivation. The vaginal epithelium subsequently becomes less cellular and thinner, and glycogen production, which is responsible for vaginal secretion, gradually declines and ultimately comes to a complete halt. Blood flow to the vagina is also reduced, which is associated with decreased fluid secretion during sexual arousal. These changes produce a variety of symptoms, and most notably, vaginal dryness, and the resultant decrease in natural lubrication increases susceptibility to trauma and pain during intercourse..$^{3-7}$

Up to two-thirds of menopausal women have physical evidence of VVA and $\sim 50 \%$ of postmenopausal women have VVA symptoms. The major findings and symptoms of VVA are decreased vaginal lubrication, leading to vaginal dryness, followed by other vaginal and urinary symptoms, such as burning, itching, bleeding, leucorrhea, dyspareunia, and dysuria. ${ }^{3,6-10}$ These symptoms usually appear $\sim 2-4$ years after the onset of menopause.

Symptoms of VVA are not limited to sexually active women with their severity ranging from mild to debilitating, and they can negatively affect patients' quality of life (QoL). 3,6,7 VVA symptoms have an adverse emotional and physical impact on patients and their partners by contributing to unsatisfactory sexual relationships. ${ }^{10-12}$

The term GSM emerged following a consensus conference held in May 2013 in consideration of the fact that VVA is also associated with a wide range of urogynecological symptoms, including urinary incontinence. ${ }^{12,13}$ Menopausal women can experience a decrease in both the diameter and quantity of periurethral striated muscles. These changes may be responsible for the observed functional effects on intrinsic and extrinsic continence mechanisms. In addition, collagen I and III content and quality at the level of the endopelvic fascia are affected and have been shown to have a close relationship with pelvic floor dysfunction, including SUI, which is the most common of the incontinence dysfunctions. ${ }^{14}$

Decreased vaginal tissue and muscular function is basically a result of damage to the vagina at a nerve, muscular, cellular, and connective tissue level caused primarily by tears and stress on the pelvic floor during pregnancy and vaginal delivery, as well as the breakdown of vaginal tissue and decreased blood flow of menopause due to estrogen deprivation. ${ }^{14-18}$ An understanding of the determinants of vaginal tissue strength and integrity is important in designing appropriate management strategies to treat GSM.
The connective tissue of the vagina is composed of fibroblasts, and smooth muscle cells, surrounded by an extracellular matrix (ECM). ${ }^{14}$ Although fibroblasts are the main cells responsible for the synthesis and secretion of fibrillar collagen and elastin and less abundant nonfibrillar components, smooth muscle cells can also synthesize these molecules. Collagen and elastin are fundamental components that control the biomechanical properties of the vaginal tissue. The ECM is constantly remodeling, and its homeostasis depends on the balance between synthesis and degradation by matrix metalloproteinases (MMPs), which are further controlled by activators and tissue inhibitors of MMPs. Both processes are modulated by soluble biological mediators, including growth factors and their receptors, as well as by chemical and mechanical signaling from the ECM, which is recognized by transmembrane receptors and integrins. ${ }^{19}$

Fibrillar collagens are the principal determinants of vaginal tissue strength. Collagen I, III, and V are the main collagen subtypes present in the vagina. Collagen $\mathrm{I}$ is abundant in skin, ligament, tendon, and bone. It forms large and strong fibers that are responsible for the mechanical resistance of tissue. Collagen III forms smaller fibers with lower tensile strength and is present in mobile organs and tissues that are cyclically stretched such as blood vessels. ${ }^{19}$ Collagen $\mathrm{V}$ forms small fibers of low tensile strength. Collagens I, III, and V copolymerize to form hybrid fibrils. Collagen $\mathrm{V}$ forms the fibril core that is surrounded by copolymers of collagen I and III. The proportion of each subtype of collagen determines the fiber size and has an impact on the biomechanical strength of the tissue. ${ }^{19}$ The collagen fibers are further covered by collagens XII and XIV and small leucine-rich proteoglycans such as decorin, which also participate in the control of fibrinogenesis. Elastic fibers are key architectural elements of connective tissues that are subject to mechanical tension and expansile forces. They provide extensibility and recoil in elastic tissues and are important in maintaining vaginal structural integrity against mechanical strain. ${ }^{18}$

GSM is unlikely to improve over time without treatment, and the signs and symptoms of GSM tend to reappear if treatment is discontinued. ${ }^{20,21}$ The main therapeutic objectives in managing GSM are to relieve genital symptoms and to attempt to restore the vaginal environment to a healthy condition. ${ }^{6}$ To-date, the most common treatments for GSM include interactive therapy using pelvic floor exercises, physical therapy, bladder training and timed canceling, pharmacologic therapy, and/or surgical intervention for those patients suffering from incontinence-related issues. Vaginal estrogens are most commonly prescribed for women suffering from VVA symptoms. Unfortunately, pelvic floor exercises have limited long-term success due to compliance issues ${ }^{22,23}$ and there are a significant number of postmenopausal women who are not candidates for use of estrogen-based creams, given a concern for increased risk as a result of previous breast or other cancers. The optimal approach for the relief of GSM symptoms needs to take into consideration patient preferences and willingness to comply with treatment regimens, in addition to being mindful of contraindications to therapy.

\section{Current Nonpharmacologic Therapies}

Various nonpharmacologic modalities have been used in the vagina and on the vulva both for therapeutic and 
recreational purposes. We discuss these in the context of their current applications and mechanism of action.

\section{Vibration}

Vibrators and self-stimulators have been applied to the vulva and vagina in a variety of forms over the past 100 years for sexual play, self-stimulation, as well as for therapeutic purposes. Vibration stimulates the vulvar and vaginal tissues, which may facilitate natural lubrication and help maintain function. ${ }^{24}$

\section{Thermal energy}

Thermal energy has been applied to the vulva and vagina in a number of forms and in a number of devices for both nontherapeutic and therapeutic reasons. Examples of some of these include the following: bathing in warm water, warm water douches, warmed intravaginal lubricants, exposure to heat lamps, use of electric stimulation incontinence devices, use of electrical vibrators, intravaginal ultrasound, and various radiofrequency (RF) and laser devices. ${ }^{24-30}$

$\mathrm{RF}, \mathrm{CO}_{2}$, and Er:YAG devices have been approved for use in the vulva and lower female genital tract. ${ }^{24-30}$ Each of these modalities produce local effects in the vaginal mucosa and subjacent tissues in part or totally via a thermal heating mechanism and, in part, by induction of heat shock proteins (HSP) and induction of a cascade of local effects. ${ }^{24-30}$

In RF-based devices, RF energy is directed to specific tissue depths to achieve and maintain temperatures in the range of $40^{\circ} \mathrm{C}-45^{\circ} \mathrm{C} .^{24,26-29}$ The delivered RF energy does not result in tissue ablation or removal as is the case with the laser technologies.

$\mathrm{CO}_{2}$ and Er:YAG devices exert their effects via specifically configured beams of light energy in the far-infrared and midinfrared spectrum, respectively. Their beam temperatures are in the range of $1500^{\circ} \mathrm{C}$, although the tissue temperature at the site of impact (zone of ablation) reaches $100^{\circ} \mathrm{C}$ due to the boiling point of water, and surrounding skin temperatures reach $120^{\circ} \mathrm{C}-200^{\circ} \mathrm{C}$ in the case of the $\mathrm{CO}_{2}$ laser. ${ }^{24-29}$ Recently, Er:YAG laser technology producing minimal ablation using $300 \mu$ s superpulsed mode with $50 \mathrm{~ms}$ intrapulse intervals and a pulse duration of $250 \mathrm{~ms}$ has been developed. ${ }^{25,27}$ This technology raises the vaginal mucosal temperature to $60^{\circ} \mathrm{C}-65^{\circ} \mathrm{C}^{25,27}$

HSP induction via thermal stress in normal tissue has not been associated with genotoxic outcomes, nor with potentiation of genotoxicity in the presence of adjuvants. Similarly, there have been no reports of abnormal cytologic or histologic changes being observed in patients who have undergone various vaginal or lower genital tract procedures with these technologies. Of particular note, $\mathrm{CO}_{2}, \mathrm{Nd}$ :YAG, and various other laser devices have been used for nearly five decades. ${ }^{27}$

Studies have demonstrated that the controlled deposition and use of thermal energy on the vaginal wall are capable of stimulating the proliferation of glycogen-enriched epithelium, neovascularization, collagen formation in the lamina propria, improvements in natural lubrication, and urinary control. ${ }^{24-29}$

\section{Photonic energy}

Light, particularly in the visible to far-infrared spectrum, has been applied to the female genital tract with lasers and other devices for nearly five decades. ${ }^{27} \mathrm{~A}$ variety of delivery devices, including free beam applications and colposcopic, microscopic, fiberoptic, and other contact probes, have been used to incise, coagulate, vaporize, and ablate tissues. These have included procedures on both normal and neoplastic tissues, and management of condylomata, endometriosis, menometrorrhagia, and other conditions. More recently, a number of fractional laser devices have been applied for the management of GSM and SUI, and to achieve so-called vaginal rejuvenation. ${ }^{24-39}$ However, it should be noted that to-date, the FDA has not specifically approved any devices for these indications. ${ }^{1,2}$ Several devices emitting in the red range of the spectrum have been used in conjunction with porphyrins and other agents for photodynamic therapy to treat various malignant or premalignant lesions. ${ }^{27}$

\section{Photobiomodulation therapy}

PBMT is being used clinically and experimentally for a variety of processes and conditions. ${ }^{39-95}$ Light sources, including lasers, light-emitting diodes (LEDs), supraluminous diodes (SLDs), and other noncoherent sources, are used both clinically and experimentally. PBMT with red and nearinfrared radiation (NIR) light has been applied clinically for a diverse array of indications, including facial rejuvenation and treatment of skin laxity, wound healing, pain management, reduction of inflammation, stimulation of hair regrowth, and treatment of acne.

Vaginal tissue, such as skin, can experience laxity as women age or go through childbirth. Microcirculation and blood flow in vaginal and urethral tissues decrease with menopause, and the decline in estrogen negatively impacting vaginal hydration and bladder function. The current rationale for treating the vaginal tissue with PBMT is to stimulate synthesis of collagen and elastin in the vaginal tissue and supporting urethrovaginal sphincter and urethra, as well as promoting vasodilation in the vaginal and urethral submucosa.

\section{Photobiomodulation mechanism of action}

The widely accepted theory is that photobiomodulation, particularly at wavelengths in the red range, activates cytochrome c oxidase and increases mitochondrial electron transport, leading to an increase in adenosine triphosphate (ATP). ${ }^{47,49-58,60,61}$ Photoacceptors in the tissue cells' mitochondria absorb red and NIR light, inducing a cascade of events resulting in the production of reactive oxygen species (ROS), nitric oxide (NO), and reactive nitrogen species.

Healing is affected by upregulation of specific substrates and inhibition of others. Collagen production is stimulated, various cytokines are upregulated [e.g., epidermal growth factor, transforming growth factor beta, and fibroblast growth factor $(\mathrm{FGF})]$, and inflammatory cytokines [e.g., interleukin (IL)-6, IL-8, and IL-1] are downregulated by photobiomodulation. Upregulation of cytochromes, other transport and energy compounds [e.g., nicotinamide adenine dinucleotide hydrogen (NADH), ATP, and adenosine diphosphate (ADP)], enhances the activities of various cellular components in the local wound milieu. ${ }^{39-48}$

Avci et al. reviewed the use of PBMT for the treatment of hair loss. ${ }^{60}$ They reported that PBMT acts on the mitochondria and may alter cell metabolism through photodissociation of inhibitory NO from cytochrome c oxidase, specifically Unit IV in the respiratory chain of mitochondria, 
causing increased ATP production, modulation of ROS, and induction of transcription factors, such as nuclear factor kappa B, and hypoxia-inducible factor-1. These transcription factors in return cause protein synthesis that triggers further effects down-stream, such as increased cell proliferation and migration, alteration in the levels of cytokines, growth factors, and inflammatory mediators, and increased tissue oxygenation. They note further that NO is known to be a potent vasodilator via its effect on cyclic guanine monophosphate production and it can be speculated that PBMT may cause photodissociation of NO not only from cytochrome c oxidase but also from intracellular stores such as nitrosylated forms of both hemoglobin and myoglobin leading to vasodilation and increased blood flow. 60

The interaction of photons with cells is a necessary and essential condition for photobiomodulation to occur. Absorption and transduction of this energy must occur and it is well known that cellular molecules and structures are capable of absorbing this energy at various wavelengths. ${ }^{45,47,49-58,60-62}$ It is also known that transmission of some portion of the incident light occurs depending on the wavelength, the irradiance, the time course of the interaction, and the particular tissue being exposed to the beam. While it is likely that a number of intracellular molecules are capable of transduction of light energy in eukaryotic cells, the cytochrome system is the primary target. Several investigators have documented that photobiomodulation upregulates ATP production via this system. ${ }^{45,47,49-58,60,61}$ However, some cells and tissues remain unresponsive to phototherapy, even when it is provided according to generally accepted and published parameters. ${ }^{60,61,67}$

Lanzafame demonstrated this phenomenon in a simple tissue model using fetal bovine heart endothelial cells (FBHE). ${ }^{67}$ These cells require growth factors for growth and proliferation. They have an absolute dependency on basic FGF (bFGF) for survival in tissue culture. ${ }^{67}$ They demonstrated that $660 \mathrm{~nm}$ photoradiation at $2.16 \mathrm{~J} / \mathrm{cm}^{2}$ significantly increased cell proliferation and bFGF production in fibroblasts, which in turn increased proliferation of FBHE. Photoirradiation of the FBHE alone did not result in changes in cell proliferation. ${ }^{67}$ Both cell lines possess mitochondria and cytochromes, but FBHE still require bFGF and are otherwise "refractory" or "unresponsive" to light exposure.

PBMT has been demonstrated to accelerate wound healing $39-46,48,54,55,74$ and reduce pain and inflammation $^{63,66,71,73}$ and cancer. ${ }^{75-79}$ PBMT augments intracellular metabolism by increasing ATP production, among other metabolic pathways, as well as to induce or reduce production of ROS and other free radicals as the mechanistic basis for the outcomes observed after using PBMT. ${ }^{43-58,63,64,66,71-73}$ The ability of PBMT to downregulate inflammation and modulate inflammatory processes via cellular metabolism and cellular responses induced by changes in ROS, NO, and other inflammatory mediators orchestrated by light exposure might also contribute to its mechanism of action. ${ }^{52-54,57,58,60-67,71,73,77}$

Work from several laboratories has demonstrated that NIR photoirradiation can accelerate wound healing and tensile strength in both normal and impaired tissues and that the effects are not related to alterations in tissue tempera- ture. ${ }^{48,68,69,74,96}$ Tissue tensile strength increases are correlated with increased collagen synthesis and tissue collagen content, demonstrated in various models and with both red and NIR wavelengths. ${ }^{48,68,69,74,96}$

\section{Safety Considerations Regarding PBMT of Vaginal Tissue}

The FDA recently issued a statement indicating that there are no energy-based devices currently marketed in the United States that have been cleared for vaginal cosmetic procedures, including indications for managing GSM, SUI, or other menopause-related conditions. ${ }^{1,2}$ The FDA statement and subsequent releases cite numerous adverse events from use of lasers and RF sources for these indications. These include both specific surgically related complications and thermal injuries causing burns, scarring, and pain. The FDA further notes that the safety and efficacy of the devices used have not been established. ${ }^{1}$

Photobiomodulation devices and therapies exert their effects via nonthermal mechanisms. PBMT has demonstrated a remarkably low incidence of adverse effects, having been used for more than 50 years for diverse medical conditions and in a variety of anatomical sites. $39-45,57-62,65,66,70-73,82,83,86-90,93$ Analysis of nonphotoradiated and photoirradiated tissues has been used to elucidate the tissue response and efficacy of the photobiomodulation effect. ${ }^{39,58,60-63,65}$ Haywood et al. found no detectable free radicals after exposure of human skin biopsy to $694 \mathrm{~nm}$ light at $11-14 \mathrm{~J} / \mathrm{cm}^{2}$ in $0.9 \mathrm{~ms}$ pulses using electron spin resonance spectroscopy. ${ }^{65}$

To the best of our knowledge, there are no reports of damage to biological tissues such as sweat glands, blood vessels, skin, muscle, or other adjacent structures by PBMT. ${ }^{39,55,64-66,75-79}$ Similarly, there is no report that these wavelengths induce genotoxic changes, mutagenesis, or cancer, even in patients undergoing chemotherapy and who are receiving PBMT for prevention and management of oral mucositis. $^{81-92}$

To-date, there has been limited use of photobiomodulation devices for PBMT of vaginal tissues. However, the use of PBMT for the management of oral mucositis and other oral conditions ${ }^{83-92}$ supports the hypothesis that use of PBMT for vaginal treatment is reasonable. Thompson demonstrated that vaginal and buccal epithelia are microscopically similar. ${ }^{84}$ They noted that there were no statistically significant differences between the maximum and minimum number of epithelial cell layers. Further, the patterns of surface keratinization and the distribution and appearance of the lipid lamellae in the intercellular spaces are similar. Thompson concluded that based on these findings, the buccal epithelium can be used as a surrogate for the vaginal epithelium. $^{84}$

Neuman and Finklestein evaluated $660 \mathrm{~nm}$ PBMT on the nasal clinical symptoms of allergic rhinitis in a doubleblinded randomized prospective study. ${ }^{89}$ A statistically significant improvement of symptoms was reported by $72 \%$ of the allergic rhinitis patients and objective improvement was endoscopically demonstrated in $70 \%$ of the patients treated by narrow-band red light illumination of the nasal mucosa at $660 \mathrm{~nm}$ when compared with the placebo group, with marked alleviation of clinical symptoms. ${ }^{89}$ 
PBMT has also been shown to be an effective treatment for other inflammatory processes in an animal model. Zigmond evaluated the effect of PBMT on mucosal healing in an acute colitis model in mice. ${ }^{85}$ PBMT was applied to the colon utilizing a small-diameter endoscope with an LEDbased light source at wavelengths of 440,660 , and $850 \mathrm{~nm}$. PBMT commenced 1 day before induction of colitis and continued during the 6-day induction period, as well as for 3-10 days thereafter. Disease activity was scored endoscopically and by histopathological assessment. A statistically significant improvement in disease severity was observed in all 3 PBMT treatment groups. ${ }^{85}$

\section{Data on the Use of PBMTs on Vaginal Tissue}

Preliminary clinical data concerning the use of devices using photonic sources operating at parameters typically used for PBMT for the treatment of vaginal tissues are discussed below. The characteristics of these devices, their existing pre-clinical and clinical data, and preliminary histologic analysis of tissue response to therapy are presented.

\section{Device characteristics}

Joylux, Inc. (Seattle, Washington) introduced PMBT as a component of devices for the treatment of vaginal tissues for GSM management under the brand names vSculpt and vFit. vSculpt and vSculpt PRO are cleared as Class IIa medical devices for SUI, dryness, and sexual dysfunction outside of the United States. The vFit and vFit PLUS devices are classified as Low Risk General Health and Wellness Devices by the FDA with claims for improvement in general vaginal wellness and sexual function. These devices apply thermal loading and photonic and vibrational energy to the vagina. The total photonic power output of the devices ranges between 1.2 and $1.4 \mathrm{~W}$, with delivered energy densities of $16-24 \mathrm{~J} / \mathrm{cm}^{2}$, depending on time and model. Each of these modalities is being used for the promotion of vaginal wellness and other therapeutic indications, using a variety of delivery devices and energy sources to achieve their desired effects. $^{24-29}$ Given the fact that the vSculpt device represents a combination product by virtue of use of vibrational, thermal, and photonic energy, it is necessary to consider the nonoptical components in the context of understanding the role of photobiomodulation as applied to vaginal tissues via this particular instrument.
The vSculpt device contains two vibration motors with six vibration mode options. Sonic technology produces vibration in the $75-110 \mathrm{~Hz}$ range. The device contains rechargeable lithium ion batteries with a power input of $5 \mathrm{~V}$, and $100 \mathrm{~mA}$ located in the external (handle) portion of the device (Fig. 1). These remain external to the vagina during use. Heat is produced by vibration and is measured to be $40^{\circ} \mathrm{C}-42^{\circ} \mathrm{C}$ at $10 \mathrm{~min}$ and $40^{\circ} \mathrm{C}-44^{\circ} \mathrm{C}$ at $12 \mathrm{~min}$ of treatment. Internal thermistors and an aluminum core heat sink are incorporated within the unit to prevent overheating during use. Most of the heat generated occurs in the section of the controls, followed by the area above the LEDs. The remaining heat that is not absorbed by the heat sink dissipates around the LEDs in the middle of the shaft of the device (Figs. 1 and 2), and less being transmited to and dissipated at the tip of the unit.

Two thermal studies were conducted to measure the temperature of vaginal tissue after vSculpt use. The first study was a pilot study using thermography to measure the temperature of the vaginal vestibule in six subjects. ${ }^{31}$ Thermography was conducted after $10 \mathrm{~min}$ of vSculpt use $(n=2)$. This was compared with thermography after $10 \mathrm{~min}$ of use of a vibrator alone $(n=2)$ or after $10 \mathrm{~min}$ of use of the ApexM device $(n=2)$, which has an FDA clearance for treatment of SUI symptoms. The vaginal vestibule temperature did not rise more than $2^{\circ} \mathrm{C}$ above baseline when measured immediately posttreatment after vSculpt, vibrator, or ApexM use.

A second institutional review board (IRB)-approved study $^{32}$ performed a temperature analysis to verify that the vSculpt device when used as indicated in human patients does not generate temperatures in excess of safe levels. Testing was conducted in 20 subjects to measure the temperature of the vSculpt device surface and the temperature of vaginal tissue during a 10-min treatment cycle. Pelvic examinations were performed by a board-certified OB/GYN pre- and posttreatment. No clinically observable changes were noted in participants' vaginal tissues posttreatment, nor were adverse events reported. The average baseline vaginal temperature was $37.3^{\circ} \mathrm{C}$, and the average posttreatment vaginal tissue temperature was $37.9^{\circ} \mathrm{C}(n=20)$. This represented an increase of $0.6^{\circ} \mathrm{C}$ over baseline on average (range, $-0.6^{\circ} \mathrm{C}$ to $2.1^{\circ} \mathrm{C}$ ). The maximum vaginal tissue temperature reached $39.2^{\circ} \mathrm{C}$ in a single patient. The average temperature at the surface of the vSculpt device after the 10min treatment was $41.2^{\circ} \mathrm{C}$ (range, $38.6^{\circ} \mathrm{C}-44.1^{\circ} \mathrm{C}$ ).
FIG. 1. The vSculpt device is demonstrated. The anterior aspect (top) of the device is shown $(\mathbf{A}, \mathbf{C})$. The posterior aspect (bottom) of the device is shown (B). The scale of the device is demonstrated (C).

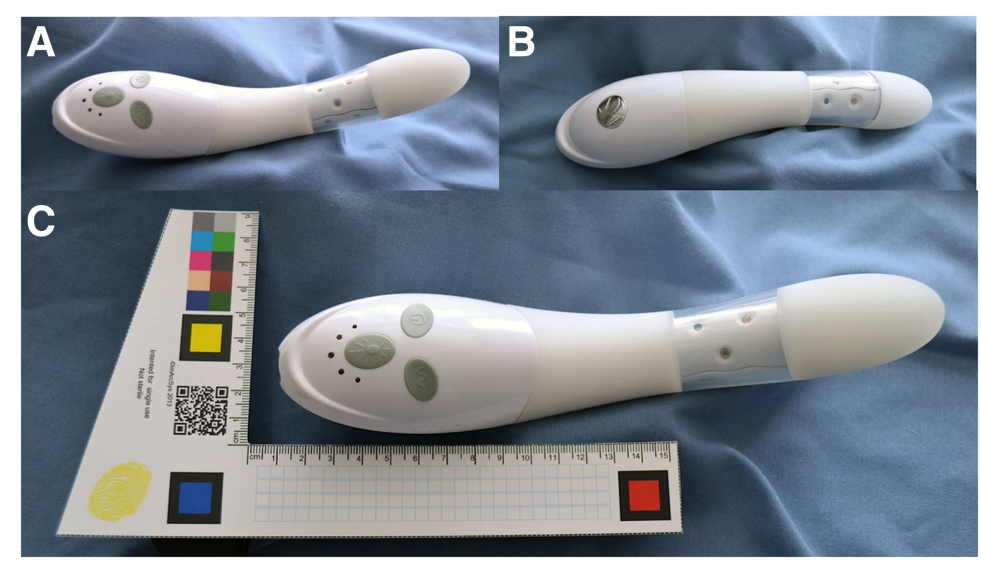




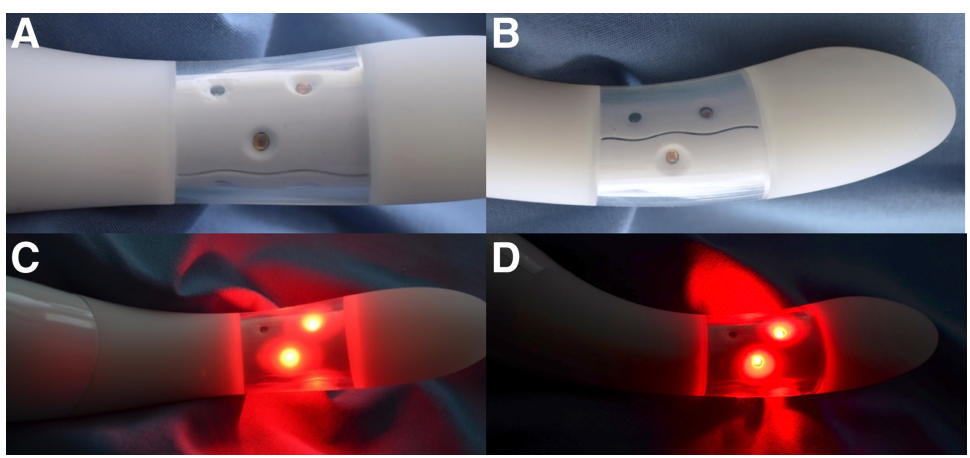

FIG. 2. The vSculpt LED source configuration and light distribution are demonstrated. Anterior device views are shown both without (A) and with device activation (C). Lateral device views are shown both without (B) and with device activation (D). LED, light-emitting diode.

This study demonstrated that the surface temperature of the vSculpt and the vaginal tissue temperature maxima after a normal treatment session are within the $40^{\circ} \mathrm{C}-45^{\circ} \mathrm{C}$ range, which is typical of RF devices such as ThermiVa and is substantially lower than the local wound temperatures of $60^{\circ} \mathrm{C}-200^{\circ} \mathrm{C}$ that occur with $\mathrm{CO}_{2}$ and Er:YAG devices. ${ }^{24-29}$

The vSculpt contains nine LEDs placed circumferentially and disposed radially within a $2.5 \mathrm{~cm}$ section of the body of the device (Figs. 1 and 2). Six LEDs emit red light $\left(662 \pm 20 \mathrm{~nm}, 15 \mathrm{~mW}, 125^{\circ}\right.$ viewing angle) and three LEDs emit NIR light $\left(855 \pm 30 \mathrm{~nm}, 15 \mathrm{~mW}, 130^{\circ}\right.$ viewing angle). The total power output of the vSculpt device is $1.4 \mathrm{~W}$, with delivered energy densities of $12 \mathrm{~J} / \mathrm{cm}^{2}$ at $6 \mathrm{~min}, 17 \mathrm{~J} / \mathrm{cm}^{2}$ at $8 \mathrm{~min}, 22 \mathrm{~J} / \mathrm{cm}^{2}$ at $10 \mathrm{~min}$, and $24 \mathrm{~J} / \mathrm{cm}^{2}$ at $12 \mathrm{~min}$. The average power density is $34 \mathrm{~mW} / \mathrm{cm}^{2}$. The location of the LEDs (Figs. 1 and 2) and their light distribution (Fig. 2C, D) are such that the photonic energy is delivered to the distal $5 \mathrm{~cm}$ of the vagina when the device is properly placed within the vaginal canal. The emitted light is directed radially and outward, rather than axially, along the shaft of the device during normal use and with normal depth of insertion of the device into the vaginal canal.

The depth of penetration of light within tissues is dependent on the incident wavelength, rather than the average power or intensity of the light source. However, a sufficient number of photons need to be applied to the tissue target to achieve an effect. The light distribution to tissue is affected by the design of the device as well as by the number and placement of optical sources within the device, the nature of the optical sources (i.e., laser, laser diode, LED, and SLD), and the distance between the device and the target or target surface. The incident beam from a laser source is generally coherent, collimated, and monochromatic (i.e., with a very narrow bandwidth). This contrasts with the light emitted from an LED source, which is neither coherent nor columnated, and these sources generally emit over a broader bandwidth, which is typically $\pm 20-30 \mathrm{~nm}$. LED sources also have a varying viewing angle, which further defines and influences the angle of divergence and attenuation of the emitted beam in both the forward and lateral directions.

The optical properties of the uterus, vagina, and adjacent structures are well studied. ${ }^{27,29,33-38}$ Bashkatov reported the depth of penetration of $660 \mathrm{~nm}$ light to be $1.6 \mathrm{~mm}$ and $850 \mathrm{~nm}$ light to be $2.25 \mathrm{~mm}$ in skin, with an associated absorption coefficient of $\sim 1.1 / \mathrm{cm} .^{10}$ The values for mucous membrane tissue were 3.5 and $5.8 \mathrm{~mm}$, respectively. ${ }^{33}$ Madsen et al. studied the depth of penetration of $630 \mathrm{~nm}$ on the uterus, noting the depth of penetration was $2.59 \pm 0.26 \mathrm{~mm}$ in postmenopausal and $4.79 \pm 0.32$ in premenopausal tissue. ${ }^{36}$ These values are consistent with the findings of Enwemeka who investigated the depth of penetration of 630 and $904 \mathrm{~nm}$ light on skin and muscle. ${ }^{34}$ Stolik studied a number of human tissues at several relevant wavelengths. ${ }^{38}$ The depth of penetration of light in the uterus was $2.40 \pm 0.22 \mathrm{~mm}$ at $675 \mathrm{~nm}$ and $3.31 \pm 0.02 \mathrm{~mm}$ at $835 \mathrm{~nm}$; muscle values were $1.63 \pm$ 0.10 and $3.72 \pm 0.29 \mathrm{~mm}$, respectively; and the depth of penetration of $675 \mathrm{~nm}$ light in the colon was $2.73 \pm 0.29 \mathrm{~mm} .^{38}$ Hardy demonstrated that vaginal therapy at $1064 \mathrm{~nm}$ was better than urethral for treating SUI. ${ }^{35}$

Based on the depth of penetration data presented above and in consideration of the rapid divergence and attenuation of light emitted from an LED source as the distance from the source increases, it is reasonable to conclude that sufficient energy would be delivered to the distal vaginal and adjacent perineal structures, but that it is highly unlikely that the delivered light would be capable of reaching the uterus during normal use and with normal depth of insertion of the device into the vaginal canal.

The red and infrared light doses delivered by the vSculpt device are at irradiances used in various PBMT regimens. The rationale for treating the vaginal tissue with light energy is based on the hypothesis that the urethra will benefit from the PBMT effect, specifically by synthesis of collagen and elastin in the vaginal tissue and the supporting urethrovaginal sphincter muscle, and promoting vasodilation in the vaginal and urethral submucosa.

\section{Preliminary clinical data}

An IRB-approved clinical study of vSculpt was conducted in the United States by de la Torre and Miller ${ }^{94}$ to measure the safety and effectiveness of the vSculpt on bladder and sexual function in women suffering from selfreported stress incontinence and sexual dysfunction issues. The protocol provided for enrollment of 55 patients at one clinical site. A sample size of 45 patients provided $90 \%$ statistical power to detect pre-to-post effect size $\geq 0.5$ with a paired $t$-test and two-sided alpha $=0.05$. A total of 55 women were enrolled in the study and 48 patients completed the study as per protocol.

Consecutive patients were evaluated for study eligibility by assessment of inclusion and exclusion criteria, medical history, and physical examination. Eligible patients were women, ages 30-59 years, with self-reported symptoms of SUI; postpartum with one or more vaginal births; painful intercourse with male partner; and/or dissatisfaction with intercourse. The main exclusion criteria were women who had an active sexually transmitted disease or infection; diabetes; 
neurological disorder; morbid obesity; current or attempted pregnancy; breastfeeding or lactating; history of cancer, chemotherapy, or radiation therapy; previous vaginal surgery or toning therapy; vesicoureteral reflux; bladder calculi or tumor; or conservative pelvic floor treatment (e.g., pelvic floor exercises and estrogen cream) in the last 6 months.

This study did not utilize a separate control group but compared patient outcomes with baseline. Given that the device emits a light that is visible and requires repeated home use, it was determined that a blinded control device (no light) would result in noncompliance treatment at home, thus invalidating the control. While patient-reported outcomes are susceptible to placebo effects, the 1-h pad weight test (PWT) is an objective standardized test that is less prone to such biases and can be evaluated against the patient's baseline. In addition, the magnitude of the treatment effects observed with urinary incontinence and sexual function questionnaire scores, as described in detail below, was greater than what might reasonably be expected due to placebo effect.

The primary efficacy end-point of the study using the FDA's Urinary Incontinence study guidance was the 1-h PWT, which is the objective measurement that most accurately measures SUI. Clinically validated QoL incontinence and sexual function questionnaires, including the Urogenital Distress Inventory-Short Form (UDI-6), the Incontinence Impact Questionnaire-Short Form (IIQ-7), the Female Sexual Function Index (FSFI), and Female Sexual Distress Scale-Revised 2005 (FSDS-R), and pelvic floor muscle strength (PFMS) using the standard Oxford Grading System were also measured as secondary efficacy end-points. Although this summary focuses on vSculpt's safety, a detailed discussion of the efficacy results was previously provided to FDA in Q170279.

The primary safety end-points were visual inspection of the vaginal tissue using the vaginal health index (VHI) scoring system by an OB/GYN practitioner and patientreported incidents of adverse events, including discomfort with device insertion or use, local tissue warmth, nerve tingling, cramping, vaginal discharge, vaginal irritation, vaginal infection, or vaginal sensitivity. Voiding diaries were also used throughout the study.

The device was used according to its proposed treatment

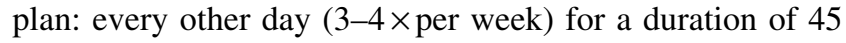
days. The examinations were performed at day 1 and at approximately day 45 , with the questionnaire being asked on days $1,14,30$, and 45 . Patients underwent $24 \pm 5$ treatments over $50 \pm 11$ days with the vSculpt device.

There were no serious adverse events reported among the women enrolled in the study. Vaginal inspection of the tissue by an OB/GYN practitioner using the VHI scoring system determined that there were no adverse changes to the vaginal tissue. Nonserious adverse events were reported by two patients (3.6\%). One patient withdrew from the study due to selfreported excessive device warmth. One urinary tract infection was reported in a patient who did not properly clean the device after each use. The patient discontinued use for 2 weeks until the infection resolved, and then resumed therapy and completed the study with no further issues. No additional reports of adverse events, including discomfort with device insertion or use, local tissue warmth, nerve tingling, cramping, vaginal discharge, vaginal irritation, vaginal infection, or vaginal sensitivity, were observed at any time during the study.

The results of this study demonstrated that multi-modal vaginal toning therapy with the vSculpt device yielded clinically meaningful improvements in bladder symptoms, PFMS, and QoL in women with SUI. It is also noted that visual inspection in all study patients by the practitioner confirmed that there were no adverse vaginal tissue changes or events to the vaginal tissue.

Gaspar and Leibaschoff recently reported the results of a preliminary study of the effects of the use of the vSculpt and controlled exercises on the perineal floor of postmenopausal women. ${ }^{95}$ Two groups of eight postmenopausal women with symptoms of vaginal dryness were recruited. All patients previously underwent laser treatment for vaginal dryness with excellent results. The PBMT group received eight treatments with the vSculpt at a frequency of two sessions weekly in the clinic along with exercises guided by a perineometer over 1 month. The control group received the exercises alone guided by the perineometer twice weekly for a total of eight treatments over the course of 1 month. Subjective improvement was evaluated by visual analog scale (VAS) scores for dyspareunia.

Biopsies were performed in six patients. Baseline biopsies were taken in the left lateral vaginal wall at the junction of the middle and lower third using Tischler Morgan biopsy forceps. Posttreatment biopsies were taken in the right lateral vaginal wall at the junction of the middle and lower third (mirror image site) using Tischler Morgan biopsy
FIG. 3. Histology from Subject 1 before (A) and 3 months after treatment (B) demonstrating restructuring of the lamina propria of the vaginal mucosa with collagenesis $(100 \times)$.
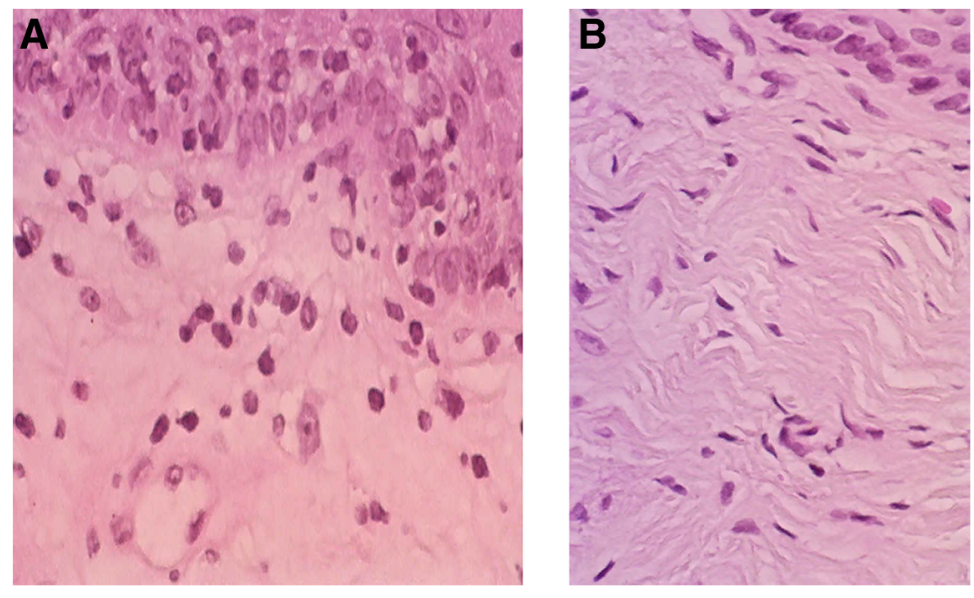
forceps. Tissues were fixed with neutral formalin, embedded in paraffin, stained with hematoxylin and eosin, and evaluated by a pathologist using standard light microscopy.

Figures 3-8 demonstrate the results. The epithelial tissue consistently exhibited parakeratosis with an increase in keratinocytes, with acanthosis and an increased glucogenic load at 3 months posttreatment. Changes were also observed in the lamina propria at 3 months posttreatment, consisting of marked angiogenesis and evidence of congestion of tissues with red cells in new vessels, collagenesis, increased cellularity of the ECM, and papillomatosis. There was no clinical or histologic evidence of thermal injury or scarring during the course of the study or at the 3-month follow-up evaluation.
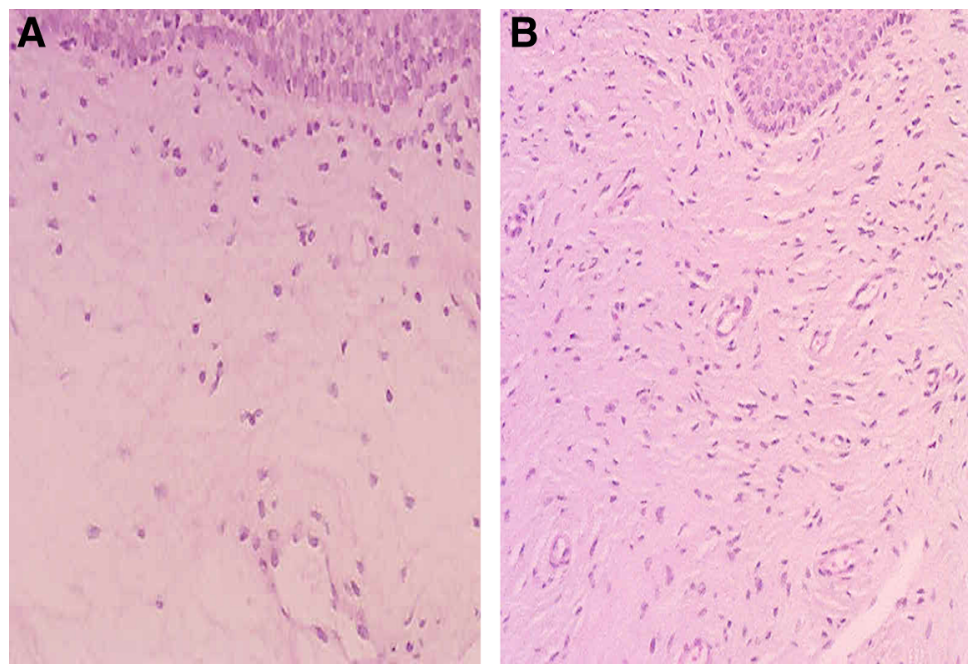

FIG. 4. Histology from Subject 2 taken at baseline (A) and 3 months (B) demonstrating increased cellularity and vascularization $(100 \times)$.
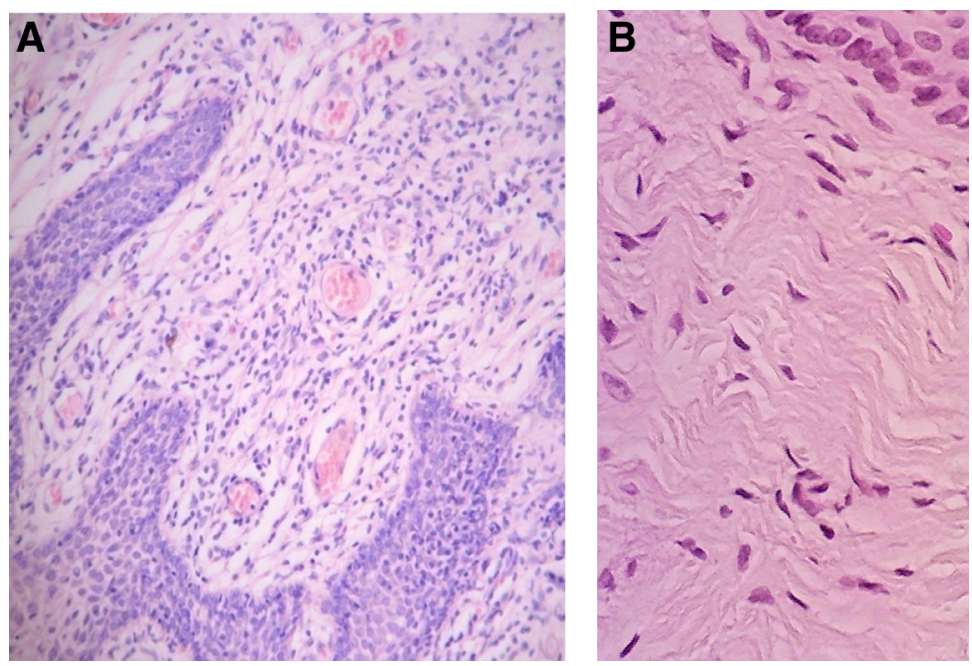

FIG. 5. Representative histology demonstrating a complete restorative reaction at the level of the ECM with increased cellularity and angiogenesis 3 months after treatment $(\mathbf{A}, 40 \times)$ and significant collagenesis $(\mathbf{B}, 100 \times)$. ECM, extracellular matrix.
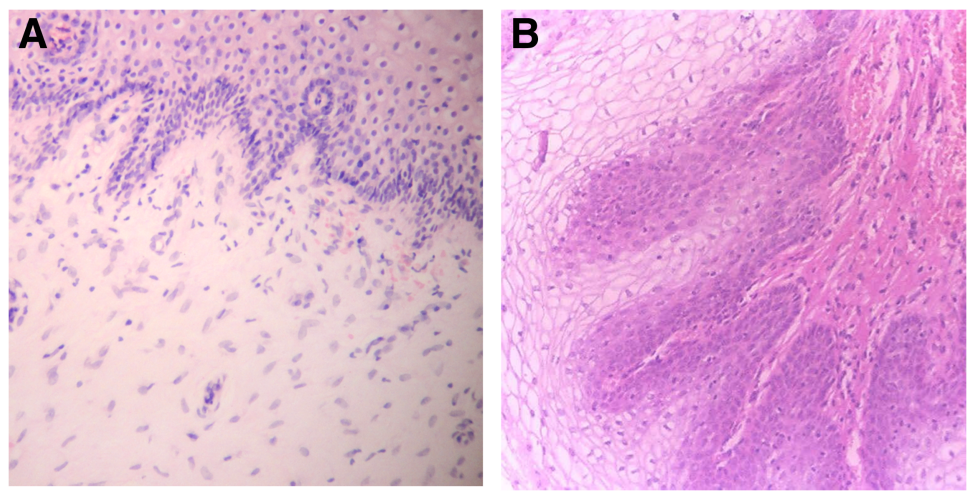

FIG. 6. Histology of Subject 3 demonstrating papillomatosis, increased glucogenic load and thickness of the vaginal epithelium, with acanthosis and parakeratosis, increased cellularity, and a complete restorative reaction at the level of the $\mathrm{ECM}$ at 3 months after treatment $(\mathbf{B}, 100 \times)$. Compared with baseline (A). 
FIG. 7. Histology of Subject 5 demonstrating noticeable changes at the level of the lamina propria from baseline (A) and at 3 months following treatment (B) with a 5-mm-thick epidermal layer $(100 \times)$. The yellow line denotes marked expansion of stratified epidermis with a thick layer of glycogenated cells and elongation of the papillae.
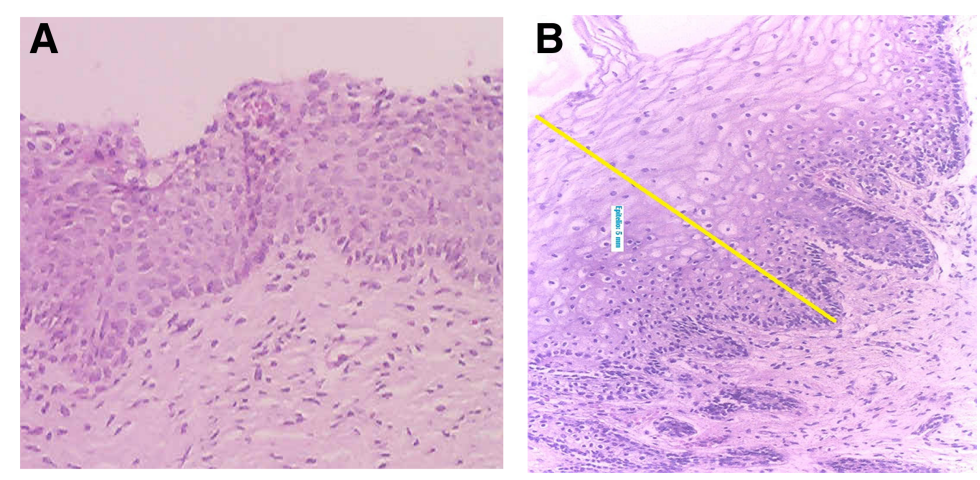

\section{Discussion}

The vSculpt is designed for self-treatment at home based on the prescription of a physician. vSculpt uses the application of gentle heat and photonic and vibrational energy to the vagina, which are technologies already used in other medical devices and have a long history of safe use. ${ }^{24-93}$ The vSculpt device is designed to help stimulate tissue healing, reduce inflammation, restore cellular function, enable biosynthesis of new collagen fibers, and promote vasodilation thereby increasing circulation to the vaginal tissue, for improved vaginal health and temporary improvement of SUI symptoms.

Both the de la Torre and Gaspar studies ${ }^{94,95}$ demonstrate that the vSculpt device is capable of producing demonstrable and sustained clinical change in postmenopausal women with GSM and/or SUI symptoms. Further, the observed findings on clinical examination and histology are consistent with those observed with the already approved RF- and light-based technologies. ${ }^{24-29}$ The Gaspar study provides evidence that the subjective measures of decreased vaginal laxity posttreatment are supported by histologic evidence of collagenesis and normalization of tissues. There is no histologic evidence of dysplasia, metaplasia, or other abnormal changes in tissue after treatment. The Iguchi ${ }^{28}$ and Hardy studies $^{35}$ in conjunction with the current understanding of the optical characteristics and absorption coefficients of the vagina, uterus, mucosa, and muscle $27,29,33-38,84$ and the design characteristics of the vSculpt provide evidence to conclude that the tissue effects of the vSculpt, like the other approved modalities, are limited to the lower genital tract and do not involve the uterus.

Pre-clinical and clinical data collected with the vSculpt confirm the intrinsic safety of the device ${ }^{31,32,94,95}$ Specifically, the available information supports the use of red and NIR light delivered at parameters commonly used in PBMT regimens for improved vaginal health and the temporary improvement of SUI symptoms. It is understood that the current technology does not represent a pure application of PBMT as monotherapy, but is a combination therapy device that also incorporates the use of vibrational and thermal energy. Certainly, there is clinical evidence of the effects of thermal modalities on vaginal tissues. However, the current data demonstrate that PBMT could conceivably improve clinical outcomes via nonthermal mechanisms, and is a reasonable modality for application to the lower female genital tract. Additional studies using the current technology and trials using PBMT alone are warranted and should be encouraged.
FIG. 8. Representative histology in one study subject $($ A $40 \times$, B $100 \times)$. The inset in (A) corresponds to the area demonstrated in the higher magnification view in (B). The vaginal mucosa is covered with a thick stratified glucogenic squamous epithelium with irregular acanthosis and papillae elongation. The basal layer is preserved and there is marked acanthosis and basal cell hyperplasia. There is mild parakeratosis and conservation of the superficial strata. The epithelium is nondysplastic and eutrophic. The chorion is edematous with foci of papillary hemorrhage, with capillary vessels and lymphatic ectasia. No signs of thermal injury are observed. Congestive vaginal mucosa is present.

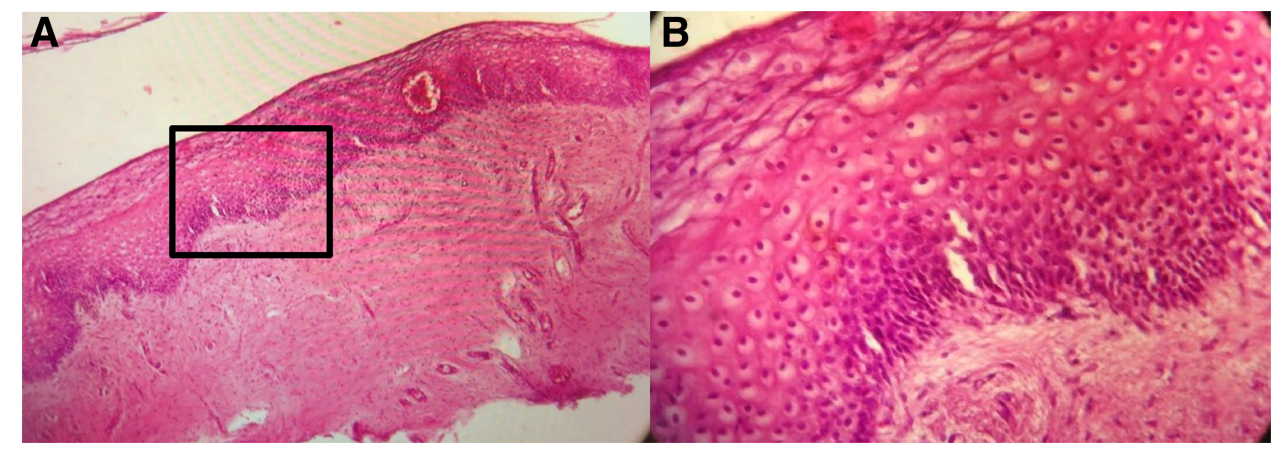




\section{Summary}

This article reviewed the biological basis, symptoms, and management of GSM and investigated the current status and rationale for the use of PBMT for improved vaginal health and the temporary improvement of SUI symptoms. Based on the preliminary evidence available, PBMT is safe and appears to be efficacious in treating GSM. These studies demonstrate that the vSculpt device is capable of producing demonstrable and sustained clinical change in postmenopausal women with GSM and/or SUI symptoms. The observed findings on clinical examination and histology are consistent with those observed with RF- and light-based technologies. While it is recognized that additional studies are warranted, PBMT represents a safe alternative in the management of these conditions.

\section{Author Disclosure Statement}

R.J.L.: Received grant funding from Apira Science; received equipment from Apira Science, ImArcSys, and LiteCure; serves as a Consultant for Joylux; holds intellectual property rights with Biomedical Gateway LLC, Conversion Energy Enterprises, ImArcSys, and New Skin Therapies LLC; serves as Officer or Director for the American Society for Laser Medicine and Surgery (CME Director), Society of Laparoendoscopic Surgeons (Executive Director/Scientific Chair); SGE Consultant General and Plastic Surgery Devices Panel of the Medical Devices Advisory Committee of the FDA CDRH; Editorial Board and Associate Editor of Lasers in Medical Science, and is Executive Editor of Photobiomodulation, Photomedicine, and Laser Surgery.

S.T.: Serves as Clinical Teaching Faculty at the University of Washington School of Medicine, Department of $\mathrm{OB} / \mathrm{GYN}$, is affiliated with Swedish Medical Center, and is the Chief Medical Officer of Joylux.

G.H.L.: Serves as General Secretary and Founder of the World Society of Cosmetic Gynecology, President of the International Union of Lipoplasty, Codirector of the University Course of Specialist in Aesthetic and Functional Gynecology and Female Aesthetic Genital Surgery University of Barcelona, Spain, Vice President of ISRAIT-The International Society of Reconstructive and Aesthetic Intimate Treatment, and is a Consultant for Joylux.

\section{References}

1. FDA Warns Against Use of Energy-Based Devices to Perform Vaginal "Rejuvenation" or Vaginal Cosmetic Procedures: FDA Safety Communication. Available at: www.fda.gov/MedicalDevices/Safety/AlertsandNotices/ ucm615013.htm (Last accessed July 30, 2018).

2. Harrison P. The quest for the perfect vagina: what you should know about cosmetic genital procedures. MedscapeAugust 27, 2018. Available at: www.medscape.com (Last accessed August 28, 2018).

3. Lev-Sagie A. Vulvar and vaginal atrophy: physiology, clinical presentation, and treatment considerations. Clin Obstet Gynecol 2015;58:476-491.

4. MacBride MB, Rhodes DJ, Shuster LT. Vulvovaginal atrophy. Mayo Clin Proc 2010;85:87-94.
5. Freedman MA. Genitourinary syndrome of menopause: physical characteristics and prevalence. Poster (\#P-21) presented at NAMS Annual Meeting, Orlando FL: October 2016. Available at: www.menopause.org/docs/default-source/ 2016-doc/scientific-poster-abstracts.pdf (Last accessed October 10, 2018).

6. Gandhi J, Chen A, Dagur G, et al. Genitourinary syndrome of menopause: an overview of clinical manifestations, pathophysiology, etiology, evaluation, and management. Am J Obstet Gynecol 2016;215:704-711.

7. Iosif CS, Batra S, Ek A, Astedt B. Estrogen receptors in the human female lower uninary tract. Am J Obstet Gynecol 1981;141:817-820.

8. Parish SJ, Nappi RE, Krychman ML, et al. Impact of vulvovaginal health on postmenopausal women: a review of surveys on symptoms of vulvovaginal atrophy. Int J Womens Health 2013;5:437-447.

9. Kingsberg SA, Wysocki S, Magnus L, Krychman ML. Vulvar and vaginal atrophy in postmenopausal women: findings from the REVIVE (Real Women's Views of Treatment Options for Menopausal Vaginal Changes) Survey. J Sex Med 2013;10:1790-1799.

10. Nappi RE, Kokot-Kierpa M. Vaginal Health: Insights, Views and Attitudes (VIVA)-results from and international survey. Climacteric 2012;15:36-44.

11. Simon JA, Kokot-Kierepa M, Goldstein J, Nappi RE. Vaginal health in the United States: results from the Vaginal Health: Insights, Views, \& Attitudes survey. Menopause 2013;20:1043-1048.

12. Nilsson M, Lalos A, Lalos O. The impact of female urinary incontinence and urgency on quality of life and partner relationship. Neurourol Urodyn 2009;28:976-981.

13. Portman DJ, Gass MLK; Vulvovaginal Atrophy Terminology Consensus Conference Panel. Genitourinary syndrome of menopause: new terminology for vulvovaginal atrophy from the International Society for the Study of Women's Sexual Health and the North American Menopause Society. Menopause 2014;21:1063-1068.

14. Rahn DD, Acevedo JF, Word RA. Effect of vaginal distention on elastic fiber synthesis and matrix degradation in the vaginal wall: potential role in the pathogenesis of pelvic organ prolapse. Am J Physiol Regul Integr Comp Physiol 2008;295:R1351-R1358.

15. Farage $M$, Maibach $H$. Lifetime changes in the vulva and vagina. Arch GYN Obstet 2006;273:195-202.

16. DeLancey JO, Morgan DM, Fenner DE, et al. Comparison of levator ani muscle defects and function in women with and without pelvic organ prolapse. Obstet Gynecol 2007; 109:295-302.

17. Ruiz-Zapata AM, Kerkhof MH, Ghazanfari S, et al. Vaginal fibroblastic cells from women with pelvic organ prolapse produce matrices with increased stiffness and collagen content. Sci Rep 2016;6:22971.

18. Liu X, Zhao Y, Pawlyk B, Damaser M, Li T. Failure of elastic fiber homeostasis leads to pelvic floor disorders. Am J Pathol 2006;168:519-528.

19. Kuhn K. In: Structure and Function of Collagen Types. R Mayne, R Burgeson (eds.). Cambridge, MA: Academic Press, 1987 p. 2.

20. Thomas HM, Bryce CL, Ness RB, Hess R. Dyspareunia is associated with decreased frequency of intercourse in the menopausal transition. Menopause 2011;18:152-157. 
21. Dennerstein L, Dudley EC, Hopper JL, Guthrie JR, Burger HG. A prospective population-based study of menopausal symptoms. Obstet Gynecol 2000;96:351-358.

22. Boyle R, Hay-Smith EJ, Cody JD, Morkved S. Pelvic floor muscle training for prevention and treatment of urinary and fecal incontinence in antenatal and postnatal women: a short version Cochrane review. Neurourol Urodyn 2014;33: 269-276.

23. Dumoulin C, Hay-Smith J, Habee-Seguin GM, Mercier J. Pelvic floor muscle training versus no treatment, or inactive control treatments, for urinary incontinence in women: a short version Cochrane systematic review with metaanalysis. Neurourol Urodyn 2015;34:300-308.

24. Faubion SS, Larkin LC, Stuenkel CA, et al. Management of genitourinary syndrome of menopause in women with or at high risk for breast cancer: consensus recommendations from the North American Menopause Society and the International Society for the Study of Women's Sexual Health. Menopause 2018;25:1-13.

25. Gaspar A, Maestri S, Silva J, et al. Intraurethral Erbium: YAG Laser for the management of urinary symptoms of Genitourinary Syndrome of Menopause: a pilot Study. Lasers Surg Med 2018;50:802-807.

26. Lang P, Karram M. Lasers for pelvic Floor dysfunctions: is there evidence? Curr Opin Obstet Gynecol 2017;29:354358.

27. Tadir Y, Gaspar A, Lev-Sagie A, et al. Light and energy based therapeutics for Genitourinary syndrome of menopause: consensus and controversies. Lasers Surg Med 2017; 49:137-159.

28. Alinsod RM. Transcutaneous temperature controlled radiofrequency for orgasmic dysfunction. Lasers Surg Med 2016;48:641-645.

29. Kwon T-R, Kim JH, Seok J, et al. Fractional $\mathrm{CO}_{2}$ laser treatment for vaginal laxity: a preclinical study. Lasers Surg Med 2018;50:940-947.

30. Iguchi M, Littmann AE, Chang SH, Wester LA, Knipper JS, Shields RK. Heat stress and cardiovascular, hormonal, and heat shock proteins in humans. J Athl Train 2012;47: 184-190.

31. JoyLux. A pilot thermographic study of vSculpt effects on the human vulva. https://www.joyluxinc.com

32. JoyLux. vSculpt clinical study, JoyLux 1515-01 thermal testing of vSculpt in Humans, Western IRB \#20160694;7/ 13/2016. https://www.joyluxinc.com

33. Bashkatov AN, Genina EA, Kochubey VI, Tuchin VI. Optical properties of human skin, subcutaneous and mucous tissues in the wavelength range from 400 to $2000 \mathrm{~nm}$. J Phys D 2005;38:2543-2555.

34. Enwemeka CS. Attenuation and penetration of visible $632.8 \mathrm{~nm}$ and invisible infra-red $904 \mathrm{~nm}$ light in soft tissues. Laser Ther 2001;13:95-101.

35. Hardy LA, Chang CH, Myers EM, Kennelly MJ, Fried NM. Computer simulations of thermal tissue remodeling during transvaginal and transurethral laser treatment of female stress urinary incontinence. Lasers Surg Med 2017;49:198205.

36. Madsen SJ, Wyss P, Svaasand LO, Haskell RC, Tadir Y, Tromberg BJ. Determination of the optical properties of the human uterus using frequency-domain photon migration and steady-state techniques. Phys Med Biol 1994;39:11911202.

37. Patterson MS, Wilson BC, Wyman DR. The propagation of optical radiation in tissue II: optical properties of tissues and resulting fluence distributions. Lasers Med Sci 1991;6: 379-390.

38. Stolik S, Delgado JA, Perez A, Anasagasti L. Measurement of the penetration depths of red and near infrared light in human "ex vivo" tissues. J Photochem Photobiol B 2000; 57:90-93.

39. Tunér J, Hode L. The New Laser Therapy Handbook. Grängesberg: Prima Books, 2011; pp. 840.

40. Hopkins JT, McLodat TA, Seegmiller JG, Baxter GD. Low-Level laser therapy facilitates superficial wound healing in humans: a triple-blind, sham-controlled study. J Athl Train 2004;39:223-229.

41. Schindl A, Schindl M, Schindl L. Successful treatment of a persistent radiation ulcer by low power laser therapy. J Am Acad Dermatol 1997;37:646-648.

42. Lanzafame RJ, Stadler I. Chapter 36: the recalcitrant wound: using low-level light therapy to manage nonhealing wounds and ulcers. In: Handbook of Low-Level Laser Therapy. MR Hamblin, T Agrawal, M De Sousa (eds.). Singapore: Pan Stanford Publishing Pte LTD., 2017; pp. 581-596.

43. Mester E, Mester AF, Mester A. The biomedical effects of laser application. Lasers Surg Med 1985;5:31-39.

44. Lam TS, Abergel RP, Castel JC, Dwyer RM, Lesavoy MA, Uitto J. Laser stimulation of collagen synthesis in human skin fibroblast cultures. Lasers Life Sci 1986;1:61-77.

45. Conlan MJ, Rapley JW, Cobb CM. Biostimulation of wound healing by low-energy laser irradiation: a review. J Clin Periodontol 1996;23:492-496.

46. Hawkins D, Houreld N, Abrahamse H. Low level laser therapy (LLLT) as an effective therapeutic modality for delayed wound healing. Ann NY Acad Sci 2005;1056:486493.

47. Passarella S, Casamassima E, Molinari S, et al. Increase of proton electrochemical potential and ATP synthesis in rat liver mitochondria irradiated in vitro by helium-neon laser. FEBS Lett 1984;175:95-99.

48. Stadler I, Lanzafame RJ, Evans R, et al. 830-nm irradiation increases the wound tensile strength in a diabetic murine model. Lasers Surg Med 2001;28:220-226.

49. Morimoto Y, Arai T, Kikuchi M, Nakajima S, Nakamura H. Effect of low-intensity argon laser irradiation on mitochondrial respiration. Lasers Surg Med 1992;15:191-199.

50. Yu W, Naim JO, McGowan M, Ippolito K, Lanzafame RJ. Photomodulation of oxidative metabolism and electron chain enzymes in rat liver mitochondria. Photochem Photobiol 1997;66:866-871.

51. Karu TI. The Science of Low Power Laser Therapy. London: Gordon and Breach Science Publishers, 1998; pp. 14 33, 53-94, 95-121.

52. Karu TI. Primary and secondary mechanisms of action of visible to near-IR radiation on cells. J Photochem Photobiol B 1998;49:1-17.

53. Vladimiorv IA, Klebanov GI, Borisenko GG, Osipov AN. Molecular and cellular mechanisms of the low intensity laser radiation effect. Biofizika 2004;49:339-350.

54. Eells JT, Wong-Riley MT, VerHoeve J, et al. Mitochondrial signal transduction in accelerated wound and retinal healing by near-infrared light therapy. Mitochondrion 2004;4:559-567.

55. Silveira PC, Streck EL, Pinho RA. Evaluation of mitochondrial respiratory chain activity in wound healing by low-level laser therapy. J Photochem Photobiol B 2007;86: 279-282. 
56. Karu TI. Low power laser therapy. In: Biomedical Photonics Handbook. T Vo-Dinh (ed.). Boca Raton, FL: CRC Press, 2003; pp. 48.1-25.

57. Liu TCY, Jiao JL, Xu XY, Liu XG, Deng SX, Liu SH. Photobiomodulation: phenomenology and its Mechanism. SPIE Proc 2004;5632:185-191.

58. Hamblin MR, Demidova TN. Mechanisms of low level light therapy. SPIE Proc 2006;6140:1-12.

59. Lanzafame RJ, Blanche RR, Chiacchierini RP, Kazmirek ER, Sklar JA. The growth of human scalp hair in females using visible red light laser and LED sources. Lasers Surg Med 2014;46:601-607.

60. Avci P, Gupta GK, Clark J, Wikonkal N, Hamblin MR. Low-level laser (light) therapy (LLLT) for treatment of hair loss. Lasers Surg Med 2014;46:144-151.

61. Huang YY, Chen ACH, Carroll JD, Hamblin MR. Biphasic dose response in low level light therapy. Dose Response 2009;7:358-383.

62. Chung H, Dai T, Sharma SK, Huang YY, Carroll JD, Hamblin MR. The nuts and bolts of low-level laser (light) therapy. Ann Biomed Eng 2012;40:516-533.

63. Meneguzzo DT, Lopes LA, Pallota R, Soares-Ferreira L, Lopes-Martins RA, Ribeiro MS. Prevention and treatment of mice paw edema by near-infrared low-level laser therapy on lymph nodes. Lasers Med Sci 2013;28:973980.

64. Wikramanayake TC, Rodriguez R, Choudhary S, et al. Effects of the Lexington LaserComb on hair regrowth in the $\mathrm{C} 3 \mathrm{H} / \mathrm{HeJ}$ mouse model of alopecia areata. Lasers Med Sci 2012;27:431-436.

65. Haywood RM, Wardman P, Gault DT, et al. Ruby laser irradiation $(694 \mathrm{~nm})$ of human skin biopsies: assessment by electron spin resonance spectroscopy of free radical production and oxidative stress during laser depilation. Photochem Photobiol 1999;70:348-352.

66. Lanzafame RJ. Photobiomodulation: an enlightened path emerges. Photomed Laser Surg 2013;31:299-300.

67. Yu W, Naim JO, Lanzafame RJ. The effect of laser irradiation on the release of bFGF from 3 T3 fibroblasts. Photochem Photobiol 1994;59:167-170.

68. Purschke M, Laubach HJ, Anderson RR, Manstein D. Thermal injury causes DNA damage and lethality in unheated surrounding cells: active thermal bystander effect. J Invest Dermatol 2010;130:86-92.

69. Purschke M, Anderson R, Zurakowski D, Manstein D. Cellcycle-dependent active thermal bystander effect (ATBE). Lasers Surg Med 2011;43:230-235.

70. Lanzafame RJ, Brondon P, Stadler I. WOUND HEALING: experimental and clinical approaches to understanding photobiomodulation and its mechanisms. In: Lasers In Medicine And Dentistry Volume 4. Z Simunovic (ed.). European Medical Laser Association. Zagreb, Croatia: AKD, 2009; pp. 163-172.

71. Chow R, Arwati P, Laakso L, Bjordal J, Baxter G. Inhibition and relevance to analgesic effects: a systematic review. Photomed Laser Surg 2009;29:365-381.

72. Chow RT, Johnson MI, Lopes-Martins RAB, Bjordal JM. Efficacy of low-level laser therapy in the management of neck pain: a systematic review and meta-analysis of randomised placebo or active-treatment controlled trials. Lancet 2009;374:1897-1908.

73. Karu T. Is it time to consider photobiomodulation as a drug equivalent? Photomed Laser Surg 2013;31:188-191.
74. Lanzafame RJ, Stadler I, Coleman J, et al. Temperaturecontrolled $830 \mathrm{~nm}$ LLLT of experimental pressure ulcers. Photomed Laser Surg 2004;22:483-488.

75. Fonseca AS, Moreira TO, Paixao DL, et al. Effect of laser therapy on DNA damage. Lasers Surg Med 2010;42:481488.

76. Myakishev-Rempel M, Stadler I, Brondon P, et al. A preliminary study of the safety of red light phototherapy of tissues harboring cancer. Photomed Laser Surg 2012;30: 551-558.

77. Stadler I, Evans R, Kolb B, et al. In vitro effects of lowlevel laser irradiation at $660 \mathrm{~nm}$ on peripheral blood lymphocytes. Lasers Surg Med 2000;27:255-261.

78. Santana-Blank L, Rodríguez-Santana E, Santana Rodríguez KE. Concurrence of emerging developments in photobiomodulation and Cancer. Photomed Laser Surg 2012;30: 615-616.

79. Santana-Blank L, Rodríguez-Santana E, Santana-Rodríguez JA, Santana-Rodríguez KE. Solid tumors and photobiomodulation: a novel approach to induce physiologically reparative homeostasis/homeokinesis-review. J Solid Tumors 2012;2:23-35.

80. Lanzafame RJ, Stadler I, Kurtz AF, et al. Reciprocity of exposure time and irradiance on energy density during photoradiation on wound healing in a murine pressure ulcer model. Lasers Surg Med 2007;39:534-542.

81. Arany PR. Healing tumors with light: science Fiction or the future of medicine? Photomed Laser Surg 2018;36:227229.

82. Hamblin MR, Nelson ST, Strahan JR. Photobiomodulation and cancer: what is the truth? Photomed Laser Surg 2018; 36:241-245.

83. Bensadoun RJ, Nair RG. Low-level laser therapy in the prevention and treatment of cancer therapy-induced mucositis: 2012 state of the art based on literature review and meta-analysis. Curr Opin Oncol 2012;24:363-370.

84. Thompson IOC, van der Bijl P. A comparative light microscopic, electron-microscopic and chemical study of human vaginal and buccal epithelium. Arch Oral Biol 2001; 46:1091-1098.

85. Zigmond E, Varol C, Kaplan M, Shapira O, Melzer E. Low-level light therapy induces mucosal healing in a murine model of dextran-sodium-sulfate induced colitis. Photomed Laser Surg 2014;32:450-457.

86. Whelan HT, Connelly JF, Hodgson BD, et al. NASA lightemitting diodes for the prevention of oral mucositis in pediatric bone marrow transplant patients. J Clin Laser Med Surg 2002;20:319-324.

87. Hodgson BD, Margolis DM, Salzman DE, et al. Amelioration of oral mucositis pain by NASA near-infrared lightemitting diodes in bone marrow transplant patients. Support Care Cancer 2012;20:1405-1415.

88. Freitas AC, Campos L, Brandão TB, et al. Chemotherapyinduced oral mucositis: effect of LED and laser phototherapy treatment protocols. Photomed Laser Surg 2014;32: 81-87.

89. Neuman I, Finkelstein Y. Narrow-band red light phototherapy in perennial allergic rhinitis and nasal polyposis. Ann Allergy Asthma Immunol 1997;78:399-406.

90. Kuhn A, Porto FA, Miraglia P, Brunetto AL. Low-level infrared laser therapy in chemotherapy-induced oral mucositis: a randomized placebo-controlled trial in children. J Pediatr Hematol Oncol 2009;31:33-37. 
91. Franca CM, França CM, Núñez SC, et al. Low-intensity red laser on the prevention and treatment of induced-oral mucositis in hamsters. Photochem Photobiol B 2009;94:25-31.

92. Wagner VP, Meurer L, Martins MA, et al. Influence of different energy densities of laser phototherapy on oral wound healing. J Biomed Opt 2013;18:128002.

93. Lee SY, Park KH, Choi JW, et al. A prospective, randomized, placebo-controlled, double-blinded, and split-face clinical study on LED phototherapy for skin rejuvenation: clinical, profilometric, histologic, ultrastructural, and biochemical evaluations and comparison of three different treatment settings. Photochem Photobiol B 2007;88:51-67.

94. de la Torre S, Miller LE. Multimodal vaginal toning for bladder symptoms and quality of life in stress urinary incontinence. Int Urogynecol J 2017;28:1201-1207.

95. Gaspar A, Leibaschoff GH. Preliminary study using a device for photobiomodulation and controlled exercises in the perineum floor. Presented at the World Society of
Cosmetic Gynecology Congress, Cartagena, Columbia, April 28, 2018.

96. Yu W, Naim JO, Lanzafame RJ. The effects of photostimulation on wound healing in diabetic mice. Lasers Surg Med 1997;20:56-63.

Address correspondence to: Raymond J. Lanzafame, MD, MBA, FACS Raymond J. Lanzafame, MD PLLC 757 Titus Avenue Rochester, NY 14617-3930

E-mail: raymond.lanzafame@gmail.com

Received: January 2, 2019. Accepted after revision: April 7, 2019. Published online: June 12, 2019. 